\title{
Modulação inflamatória em equinos após artroscopia e aplicação intra-articular de plasma rico em plaquetas, de pentorse ou de acetonido de triancinolona
}

Miguel Gallio ${ }^{[]^{*}}$, Heloisa Einloft Palma ${ }^{[a]}$, Flávio De La Côrte ${ }^{[a]}, K^{2}$ arin Érica Brass ${ }^{[a]}$, Luis Felipe Dias Lopes ${ }^{[a]}$, Marta Maria Medeiros Frescura Duarte ${ }^{[a]}$, Roberta Carneiro da fontoura Pereira ${ }^{[b]}$

\footnotetext{
[a] Universidade Federal de Santa Maria (UFSM), Santa Maria, RS, Brasil

[b] Universidade Regional do Noroeste do Estado do Rio Grande do Sul (UNIJUÍ), Unijuí, RS, Brasil
}

*Autor correspondente

e-mail:vetgallio@yahoo.com.br

\section{Resumo}

O plasma rico em plaquetas (PRP) e o pentosan polissulfato de sódio (NaPPS) são dois representantes de novas terapias utilizadas com o objetivo de melhorar o ambiente articular após eventos traumáticos. 0 objetivo deste trabalho foi avaliar a modulação inflamatória de articulações submetidas à cirurgia artroscópica, tratadas com acetonido de triancinolona (TA) ou com PRP ou com NaPPS. As articulações que apresentavam fraturas osteocondrais nos ossos do carpo e osteocondrose na articulação tíbio-társica, confirmadas através do exame radiográfico, foram selecionadas. Os animais passaram por um período de descanso de 15 dias, sem o uso de anti-inflamatórios ou outras medicações. 0 procedimento consistiu na inspeção articular e retirada de todos os fragmentos ósseos como de rotina, seguido pela curetagem do leito da fratura até exposição do osso subcondral saudável. As amostras de líquido sinovial foram coletadas em quatro momentos, sendo o $1^{\circ}$ imediatamente antes de se iniciar o procedimento cirúrgico, o $2^{2}$ aos 30 dias de pós-cirúrgico (imediatamente antes da realização do primeiro tratamento), o $3^{\circ}$ aos 45 dias de póscirúrgico (imediatamente antes da realização do segundo tratamento) e o 4oa aos 60 dias de pós-cirúrgico. Após a coleta, as amostras foram acondicionadas em tubo eppendorf estéril sem anticoagulante, submetidas à centrifugação e o sobrenadante foi congelado até o momento da análise. 0 PRP foi produzido pelo método manual, a partir do sangue total colhido da veia jugular dos animais. O TA e o NaPPS foram adquiridos através da compra ou doação de produto comercial. Nove articulações foram tratadas com três mililitros de PRP (363.605 \pm 150.237 plaquetas $/ \mu \mathrm{L}$ ), nove articulações tratadas com $250 \mathrm{mg}$ de NaPPS e sete articulações tratadas com 6mg de TA. As medições das interleucinas (IL)-1, IL-6, IL-10 e do fator de necrose tumoral $\alpha$ $(\mathrm{TNF} \alpha$ ) foram efetuadas por meio de kits comerciais ELISA. Os dados foram comparados dentro do grupo a que pertenciam utilizando-se os testes ANOVA de medidas repetidas e de diferenças mínimas significativas 
de Tukey com significância estabelecida de $\mathrm{P} \leq 0,05$. Foram observadas reduções significativas nos níveis da IL-1 ( $\mathrm{P}=0,001)$, IL-6 ( $\mathrm{P}=0,004)$ e do TNF $\alpha(\mathrm{P}=0,003)$, ao mesmo tempo em que o nível da IL-10 $(\mathrm{P}=0,02)$ aumentou após a segunda aplicação do PRP. O NaPPS também esteve associado à redução dos níveis das citocinas IL-1 ( $\mathrm{P}=0,03)$ e IL-6 ( $\mathrm{P}=0,04)$, porém não esteve associado à diminuição do TNF $\alpha$. Ainda houve aumento significativo do nível da IL-10 ( P = 0,008) associado ao seu uso. Neste estudo, após o tratamento das articulações com TA, não foi observado diferença entre os níveis das citocinas pró-inflamatórias IL-1, IL-6 e nem do TNF $\alpha$, porém houve aumento na concentração da IL-10 ( $\mathrm{P}=0,003)$ associado ao uso de TA. Este resultado sugere que a aplicação de $6 \mathrm{mg}$ de TA possa apresentar modulação inflamatória, porém em menor grau do que o provido pelas outras terapias aqui empregadas. A modulação inflamatória no líquido sinovial foi influenciada positivamente por todas as terapias. O PRP, um tratamento biológico autólogo, apresentou os melhores resultados, seguido pelo NaPPS e após o TA, sendo seu uso viável após as cirurgias artroscópicas em equinos.

Palavras-chave: Citocinas. Fraturas articulares. Osteocondrose. 